\title{
Scrub Typhus Outbreak in Chonburi Province, Central Thailand, 2013
}

\author{
Wuttikon Rodkvamtook, Narupon Kuttasingkee, \\ Piyada Linsuwanon, Yutthapong Sudsawat, \\ Allen L. Richards, Maneerat Somsri, \\ Noppadon Sangjun, Chien-Chung Chao, \\ Silas Davidson, Elizabeth Wanja, \\ Jariyanart Gaywee
}

Investigation of a scrub typhus outbreak in Thailand during September 2013 found that $9.1 \%$ of Thai soldiers and $11.1 \%$ of residents living in areas surrounding training sites had antibodies against the causative agent, Orientia tsutsugamushi. Sequence analysis of $O$. tsutsugamushi from rodents and chiggers identified 7 genogroups and 3 genotypes.

\begin{abstract}
A lthough scrub typhus is one of the leading causes of acute febrile illnesses (AFI) in the Asia-Pacific region, with an estimated 1 million cases annually $(1,2)$, its overall molecular epidemiology remains unclear. Scrub typhus occurs when humans are bitten by parasitic larvae of trombiculid mites (chiggers) harboring the bacterium Orientia tsutsugamushi. Chiggers primarily infest mammals, such as rodents, that dwell in tall grasses and scrub vegetation; thus, activities such as camping and hiking can lead to scrub typhus $(3,4)$. Although scrub typhus is treatable with doxycycline if diagnosed early, the median fatality rate among scrub typhus patients is $6 \%$, and the fatality rate may reach $20 \%$ among untreated patients $(5,6)$. No effective vaccine is currently available because of high variation in $O$. tsutsugamushi immunogenicity $(7,8)$.
\end{abstract}

Scrub typhus outbreaks are problematic for the Royal Thai Army (RTA), particularly troops conducting field training in central and northeastern regions of Thailand. In a 2002 scrub typhus outbreak reported among RTA soldiers who trained in fields located in Bo Thong District, Chonburi Province, $9.8 \%$ of exposed soldiers showed seropositivity to scrub typhus group (STG) antigens (9). In 2013, an unusual pattern of AFI emerged among soldiers

Author affiliations: Royal Thai Army Component-Armed Forces Research Institute of Medical Science, Bangkok, Thailand (W. Rodkvamtook, N. Kuttasingkee, Y. Sudsawat, M. Somsri, N. Sangjun, J. Gaywee); US Army Medical Directorate-Armed Forces Research Institute of Medical Science, Bangkok (P. Linsuwanon, S. Davidson, E. Wanja); Naval Medical Research Center, Silver Spring, Maryland, USA (A.L. Richards, C.-C. Chao)

DOI: https://doi.org/10.3201/eid2402.171172 who conducted military operations at Si Racha, $89 \mathrm{~km}$ from Bo Thong. We describe the scrub typhus outbreak among RTA soldiers in 2013 and demonstrate successful integration of a control program designed with the Armed Forces Research Institute of Medical Science (AFRIMS), the AFRIMS-Nawamintharachini model, to provide more accurate assessment of potential health risks to soldiers conducting training exercises in scrub typhus-endemic areas.

\section{The Study}

During September 2013, a group of 110 RTA soldiers conducted operational field training near the Bang Phra Navy Agriculture Center in Si Racha (Figure 1). Two to 3 weeks after training, 10 soldiers experienced sudden onset of high-grade fever with clinical symptoms including intense headache, chill, and retroorbital eye pain. Eight of these soldiers were hospitalized at Fort Nawamintharachini Hospital, and the other 2 were admitted to other hospitals in Chonburi. Physical examinations revealed that $37.5 \%$ had necrotic lesions similar to eschar. Serum samples were send to AFRIMS for diagnostic tests for the most likely pathogens: PCR for dengue viruses and indirect immunofluorescent assay and dot ELISA for rickettsioses $(10,11)$ (Table 1).

Serologic analysis showed that all patients had positive antibody titers against STG, suggesting active infection. Patients were treated with oral doxycycline, and fever subsided within $24 \mathrm{~h}$ without any sequelae. For the 2 soldiers admitted at different hospitals, the serum samples from the acute and convalescent phases were not available for the assay but were collected for follow-up analysis. Serologic analysis suggested that these soldiers had higher titers of STG IgM and $\mathrm{IgG}$, indicating history of $O$. tsutsugamushi infection.

To determine the exposure of the troops to disease vectors in the training areas, we identified the serostatus of 100 additional RTA soldiers who conducted field training exercises in the same areas and of 27 residents and workers in the agriculture center. All 127 participants were healthy and afebrile at the time of blood collection. Serum tests of the residents and workers revealed that $3 / 27$ had STG IgG, suggesting high exposure to $O$. tsutsugamushi. According to interviews, 24 soldiers reported experiencing AFI during training. Test results demonstrated that none of these soldiers had $O$. tsutsugamushi infection during the training period. We concluded that seroprevalence of STG antigens among the troops was $9.1 \%$ and among residents, $11.1 \%$. 


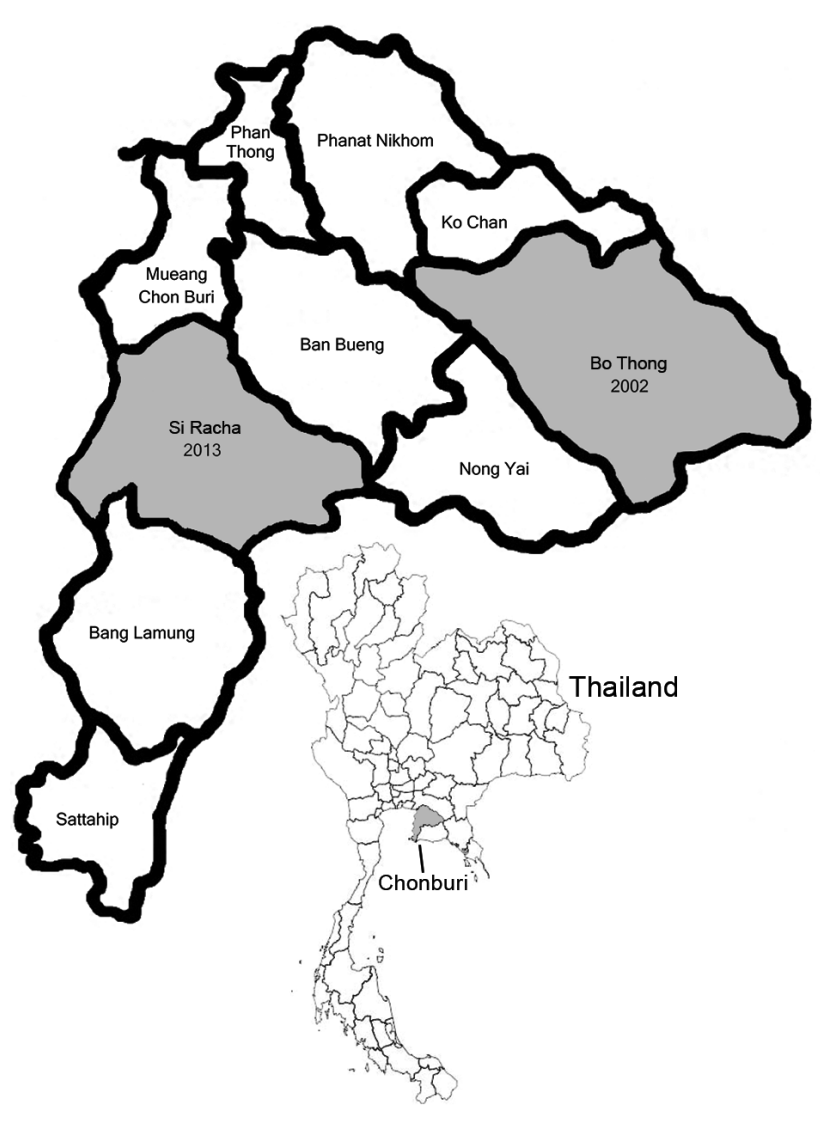

Figure 1. Chonburi Province, Thailand, showing scrub typhus outbreak areas in in Bo Thong district in 2002 and Sri Racha district in 2013 (gray shading). Inset shows location of Chonburi Province in Thailand.
To control the scrub typhus outbreak, AFRIMS applied an established prevention and control program, the AFRIMS-Nawamintharachini model, which was established in 2003 after a scrub typhus outbreak in Bo Thong District. The model describes controlling scrub typhus outbreaks through a surveillance program to clarify vector distribution and rodent population density. The program included developing diagnostic methods, increasing scrub typhus infection awareness, and providing topical insect repellents to soldiers who conduct activities in locations likely to harbor scrub typhus, as well as observing soldiers for 15 days after completing deployment for indications of AFI. We introduced this program in Si Racha after the 2013 outbreak, and since then, no scrub typhus infection has been reported in postdeployment soldiers who trained in previously defined scrub typhus risk areas.

Because rodents influence mite population density $(12,13)$, we surveyed for chiggers and small rodents in these areas to determine the etiology associated with scrub typhus outbreak and evaluate the effectiveness of the prevention program. We conducted surveys in 3 consecutive years (August 2014, November 2015, and August 2016) by following a previously described protocol (10). We surveyed areas covering a $10-\mathrm{km}$ radius near training sites with small villages and various vegetation characteristics. We collected rodent liver, spleen, and serum specimens and stored them in liquid nitrogen. We removed chiggers from rodents' ears and preserved them in $70 \%$ ethanol. We slide-mounted approximately one fifth of chigger samples from each rodent for species identification and

\begin{tabular}{|c|c|c|c|c|c|c|c|c|c|c|c|c|c|}
\hline \multirow[b]{3}{*}{ ID } & \multirow[b]{3}{*}{ Age, y } & \multicolumn{5}{|c|}{ Clinical signs and symptoms } & \multirow{3}{*}{$\begin{array}{c}\text { Symptom } \\
\text { onset to } \\
\text { collection, d† }\end{array}$} & \multicolumn{6}{|c|}{ Test results for O. tsutsugamushi, IgM/lgG } \\
\hline & & \multirow[b]{2}{*}{ Fever } & \multirow[b]{2}{*}{ Eschar } & \multirow[b]{2}{*}{ Headache } & \multirow[b]{2}{*}{ Chill } & \multirow{2}{*}{$\begin{array}{l}\text { Eye } \\
\text { pain }\end{array}$} & & \multicolumn{3}{|c|}{ IFA } & \multicolumn{3}{|c|}{ Dot-ELISA } \\
\hline & & & & & & & & 1 & 2 & 3 & 1 & 2 & 3 \\
\hline P01 & 25 & + & + & + & + & + & 3 & $\mathrm{Neg} / \mathrm{Neg}$ & $\begin{array}{l}1,600 / \\
1,600\end{array}$ & $\begin{array}{l}1,600 / \\
1,600\end{array}$ & $-1+$ & $+/+$ & $+/+$ \\
\hline P02 & 22 & + & - & + & + & - & 5 & $200 / 50$ & $\begin{array}{c}1,600 / \\
50\end{array}$ & $\begin{array}{c}1,600 / \\
50\end{array}$ & $+/+$ & $+/+$ & $+/+$ \\
\hline P03 & 25 & + & + & + & + & - & 5 & $200 / 200$ & $\begin{array}{l}1,600 / \\
3,200\end{array}$ & $\begin{array}{l}1,600 / \\
3,200\end{array}$ & $+/+$ & $+/+$ & $+/+$ \\
\hline P04 & 23 & + & - & + & + & - & 5 & $200 / 100$ & $\begin{array}{c}3,200 / \\
800\end{array}$ & $\begin{array}{c}3,200 / \\
800\end{array}$ & $+/+$ & $+/+$ & $+/+$ \\
\hline P05 & 21 & + & - & + & + & - & 5 & $200 / 100$ & $\begin{array}{c}3,200 / \\
800\end{array}$ & $\begin{array}{c}3,200 / \\
800\end{array}$ & $+/+$ & $+/+$ & $+/+$ \\
\hline P06 & 21 & + & - & + & + & + & 5 & $800 / 100$ & $\begin{array}{l}6,400 / \\
3,200\end{array}$ & $\begin{array}{l}6,400 / \\
3,200\end{array}$ & $+/+$ & $+/+$ & $+/+$ \\
\hline P07 & 21 & + & - & + & - & - & 8 & ND & $\begin{array}{c}1,600 / \\
100\end{array}$ & $\begin{array}{l}6,400 / \\
6,400\end{array}$ & ND & $+/+$ & $+/+$ \\
\hline P08 & 22 & + & - & - & + & - & 9 & ND & $800 / 50$ & $800 / 50$ & ND & $+/+$ & $+/+$ \\
\hline P09 & 45 & + & - & + & - & - & 23 & ND & ND & $\begin{array}{c}1,600 / \\
1,600\end{array}$ & ND & ND & $+/+$ \\
\hline P10 & 21 & + & + & + & + & + & 12 & ND & ND & $\begin{array}{l}3,200 / \\
3,200\end{array}$ & ND & ND & $+/+$ \\
\hline
\end{tabular}

${ }^{*}$ ID, patient identification number; IFA, indirect fluorescence antibody assay; ND, not determined; + positive; -, negative.

†Time from onset of signs and symptoms to first blood collection.

łKarp, Kato, and Gilliam genotypes. Tests were performed on September 27 (round 1), October 4 (round 2), and October 7 (round 3). Positivity cutoff titer for initial screening was 1:50 and the diagnostic criteria were established as a $>4$-fold increase in the titer of IgM or IgG in paired serum samples. If only 1 serum sample was available, titer of 1:50 to $<1: 400$ was determined as recent or previous infection and titer $\geq 1: 400$ as active infection. 
subjected the remaining chiggers to nucleic acid extraction. We extracted nucleic acid from chiggers and rodent liver and spleen samples using GeneJET Viral DNA/RNA Purification Kit (Invitrogen, Waltham, Massachusetts, USA) and assayed samples for $O$. tsutsugamushi DNA using 47-kDa quantitative PCR and 56-kDa nested PCR (14). We bidirectionally sequenced partial sequences of 56-kDa genes (First BASE Laboratories, Singapore) and used BioEdit software (http://bioedit.software.informer. com/7.1/) with the ClustalW algorithm to align nucleotide sequences with the sequences of $O$. tsutsugamush $i$ prototypes and variants retrieved from GenBank. We constructed phylogenetic trees using MEGA software (http://www.megasoftware.net/) (Figure 2).

We captured a total of 45 rodents, accounting for a $7 \%$ capture rate. Bandicota $(\mathrm{n}=5)$ and Rattus $(\mathrm{n}=30)$ spp. rats were the most commonly trapped small mammals $(77.8 \%)$, followed by ground squirrels (Menetes berdmorei, 17.8\%) and tree shrews (Tupaia glis, 4.4\%) (Table 2). Of the small mammals collected, $71.1 \%(32 / 45)$ tested STG seropositive. Most ectoparasites collected from rodents were chigger mites $(88.9 \%)$, with Leptotrombidium deliense $(72.1 \%)$ the predominant species of the 400 individual chiggers identified. A total of $20 \%$ of rodent liver and spleen samples and

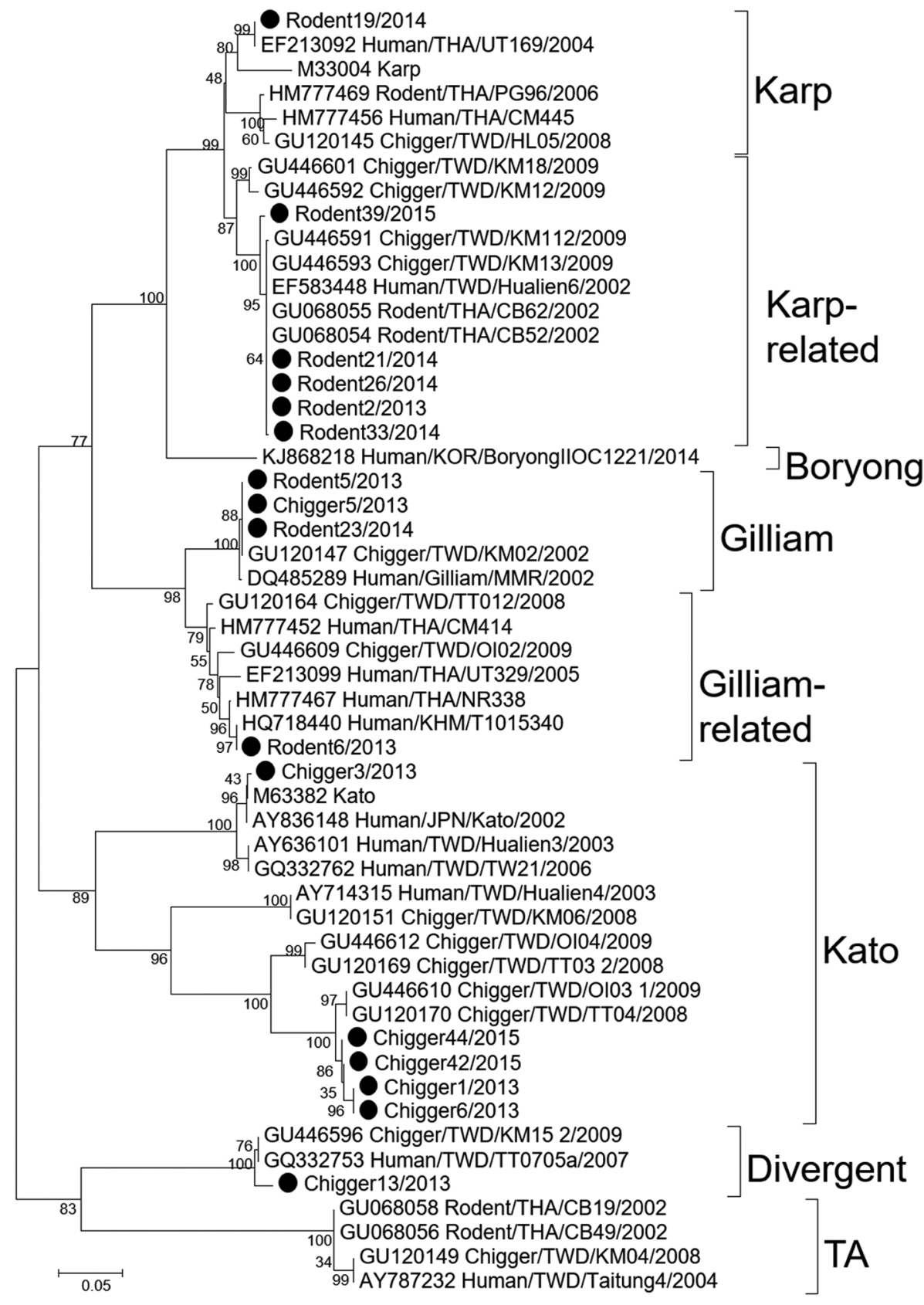

Figure 2. Phylogenetic tree of nucleotide sequences of partial Orientia tsutsugamushi $56-\mathrm{kDa}$ type-specific antigen encoding genes obtained from rodents and chiggers in Chonburi Province, Thailand, 2013 (black circles). Tree was constructed by neighbor-joining on the basis of the Kimura 2-parameter model and maximum-likelihood methods using the general time-reversible model. Bootstrapping for 1,000 replications was included in all phylogenetic tree constructions. No difference in tree topology was observed among phylogenetic trees constructed by the 2 methods. Nucleotide sequences of chigger and rodent tissue samples from Thailand have been deposited in GenBank (accession nos. MF431253-MF431268); GenBank accession numbers are provided for reference sequences. Genotypes are indicated at right. Scale bar indicates nucleotide substitutions per site. 
Table 2. Chigger infestation and Orientia tsutsugamushi infection in rodent and shrew species, Chonburi Province, Thailand, 2013*

\begin{tabular}{llcccc}
\hline Family & Species & $\begin{array}{c}\text { No. captured } \\
\text { (\% of total) }\end{array}$ & $\begin{array}{c}\text { No. infested with chiggers, } \\
\text { (\% of species total) }\end{array}$ & $\begin{array}{c}\text { No. (\%) seropositive } \\
\text { for STG by IFA }\end{array}$ & $\begin{array}{c}\text { No. (\%) positive } \\
\text { for OT by PCR }\end{array}$ \\
\hline Muridae & Bandicota indica rat & $4(8.9)$ & $4(100)$ & $4(100)$ & 0 \\
& B. savilei rat & $1(2.2)$ & $1(100)$ & 0 & $1(100)$ \\
& Rattus rattus rat & $27(60)$ & $25(92.6)$ & $27(100)$ & $7(25.9)$ \\
& R. exulans rat & $3(6.7)$ & $1(33.3)$ & $1(33.3)$ & ND \\
\hline Sciuridae & Menetes berdmorei squirrel & $8(17.8)$ & $7(87.5)$ & ND & $1(16.3)$ \\
\hline Tupaiidae & Tupaia glis shrew & $2(4.4)$ & $2(100)$ & $32(71.1)$ & 10 \\
\hline All & & 45 & $40(88.9)$ & $(22.2)$ \\
\hline${ }^{*}$ IFA, indirect fluorescence antibody assay; ND, not determined; OT, Orientia tsutsugamushi; STG, scrub typhus group of antigens.
\end{tabular}

$15.6 \%$ of individual chiggers were PCR positive for $O$. $t s u$ tsugamushi. Phylogenetic analyses of the $56-\mathrm{kDa}$ genes of 9 rodent and 7 chigger samples suggested that sequences were clustered in 3 genogroups and were closely related to 3 genotypes (Karp-related, Kato-related, and Gilliam-related genotypes) with $92.6 \%-99.8 \%$ nucleotide identity with reference sequences (Figure 2). Variants from the same clusters showed $94 \%-98 \%$ nucleotide identity with each other. Additionally, phylogenetic trees suggested that $O$. tsutsugamushi strains recovered from chiggers differed from those of rodent hosts, indicating high genetic variation of $O$. tsutsugamushi strains circulating in the survey areas.

\section{Conclusions}

High seropositive status for $O$. tsutsugamushi among RTA soldiers and the presence of disease-carrying vectors and rodent hosts trapped in training and surrounding areas indicate that soldiers in these areas are at risk of scrub typhus, with the infection rate estimated at $9 \%-11 \%$. Military personnel and residents who participate in exercises or travel to these areas in Chonburi should be alerted to the risk for scrub typhus. Appropriate protection, such as proper use of treated uniforms and application of topical insect repellent, are recommended to prevent chigger bites. Environment modification, including vegetation cleanup and trash consolidation, is necessary to control rodent and chigger populations in training location and decrease disease transmission.

RTA-AFRIMS and the Association of Military Surgeons of Thailand under the Royal Patronage of His Majesty the King provided financial support for this project. This research was supported in part by the Global Emerging Infections Surveillance and Response System (GEIS), a division of the Armed Forces Health Surveillance Branch to the Department of Entomology, USAMD-AFRIMS, with support to A.L.R. under work unit number 0000188M.0931.001.A0074. We also thank the soldiers of the RTA for their participation.

Material has been reviewed by the Walter Reed Army Institute of Research. There is no objection to its presentation and/or publication. The opinions or assertions contained herein are the private views of the authors, and are not to be construed as official, or as reflecting true views of the Department of the Army or the Department of Defense. A.L.R. and C.-C. C. are employees of the U.S. Government. This work was prepared as part of their official duties. Title 17 U.S.C. $\$ 105$ provides that Copyright protection under this title is not available for any work of the United States Government. Title 17 U.S.C. §101 defines a U.S. Government work as a work prepared by military service member or employee of the U.S. Government as part of that person's official duties.

\section{About the Author}

Dr. Rodkvamtook, a colonel in the Royal Thai Army, works as the Deputy Director of the Research Division, RTA-AFRIMS, Bangkok. His research projects include epidemiology of scrub typhus and rickettsiae, chiggers in Thailand, and diagnostic test kit development for rickettsiosis.

\section{References}

1. Watt G, Parola P. Scrub typhus and tropical rickettsioses. Curr Opin Infect Dis. 2003;16:429-36. http://dx.doi.org/10.1097/ 00001432-200310000-00009

2. Vallée J, Thaojaikong T, Moore CE, Phetsouvanh R, Richards AL, Souris M, et al. Contrasting spatial distribution and risk factors for past infection with scrub typhus and murine typhus in Vientiane City, Lao PDR. PLoS Negl Trop Dis. 2010;4:e909. http://dx.doi.org/10.1371/journal.pntd.0000909

3. Watt G, Strickman D. Life-threatening scrub typhus in a traveler returning from Thailand. Clin Infect Dis. 1994;18:624-6. http://dx.doi.org/10.1093/clinids/18.4.624

4. Nachega JB, Bottieau E, Zech F, Van Gompel A. Travel-acquired scrub typhus: emphasis on the differential diagnosis, treatment, and prevention strategies. J Travel Med. 2007;14:352-5. http://dx.doi.org/10.1111/j.1708-8305.2007.00151.x

5. Taylor AJ, Paris DH, Newton PN. A systematic review of mortality from untreated scrub typhus (Orientia tsutsugamushi). PLoS Negl Trop Dis. 2015;9:e0003971. http://dx.doi.org/10.1371/ journal.pntd.0003971

6. Khan SA, Bora T, Laskar B, Khan AM, Dutta P. Scrub typhus leading to acute encephalitis syndrome, Assam, India. Emerg Infect Dis. 2017;23:148-50. http://dx.doi.org/10.3201/eid2301.161038

7. Valbuena G, Walker DH. Approaches to vaccines against Orientia tsutsugamushi. Front Cell Infect Microbiol. 2013;2:170. http://dx.doi.org/10.3389/fcimb.2012.00170

8. Chattopadhyay S, Richards AL. Scrub typhus vaccines: past history and recent developments. Hum Vaccin. 2007;3:73-80. http://dx.doi.org/10.4161/hv.3.3.4009

9. Ruang-Areerate T, Jeamwattanalert P, Rodkvamtook W, Richards AL, Sunyakumthorn P, Gaywee J. Genotype diversity and distribution of Orientia tsutsugamushi causing scrub typhus in Thailand. J Clin Microbiol. 2011;49:2584-9. http://dx.doi.org/ 10.1128/JCM.00355-11 
10. Rodkvamtook W, Ruang-Areerate T, Gaywee J, Richards AL, Jeamwattanalert $\mathrm{P}$, Bodhidatta $\mathrm{D}$, et al. Isolation and characterization of Orientia tsutsugamushi from rodents captured following a scrub typhus outbreak at a military training base, Bothong district, Chonburi province, central Thailand. Am J Trop Med Hyg. 2011;84:599-607. http://dx.doi.org/10.4269/ ajtmh.2011.09-0768

11. Rodkvamtook W, Zhang Z, Chao CC, Huber E, Bodhidatta D, Gaywee J, et al. Dot-ELISA rapid test using recombinant 56-kDa protein antigens for serodiagnosis of scrub typhus. Am J Trop Med Hyg. 2015;92:967-71. http://dx.doi.org/10.4269/ ajtmh.14-0627

12. Kim HC, Lee IY, Chong ST, Richards AL, Gu SH, Song JW, et al. Serosurveillance of scrub typhus in small mammals collected from military training sites near the DMZ, Northern Gyeonggi-do, Korea, and analysis of the relative abundance of chiggers from mammals examined. Korean J Parasitol. 2010;48:237-43. http://dx.doi.org/10.3347/kjp.2010.48.3.237

13. Wei Y, Huang Y, Li X, Ma Y, Tao X, Wu X, et al. Climate variability, animal reservoir and transmission of scrub typhus in southern China (version 2) PLoS Negl Trop Dis.

2017;11:e0005447. http://dx.doi.org/10.1371/journal.pntd.0005447

14. Jiang J, Chan TC, Temenak JJ, Dasch GA, Ching WM, Richards AL. Development of a quantitative real-time polymerase chain reaction assay specific for Orientia tsutsugamushi. Am J Trop Med Hyg. 2004;70:351-6.

Address for correspondence: Wuttikon Rodkvamtook, Armed Forces Research Institute of Medical Sciences (AFRIMS), Royal Thai Army Component, 315/6 Rajvithi Rd, Rajthevee, Bangkok 10400, Thailand; email: rwuttikon@gmail.com, ltwutti@hotmail.com

\section{The Public Health Image Library (PHIL)
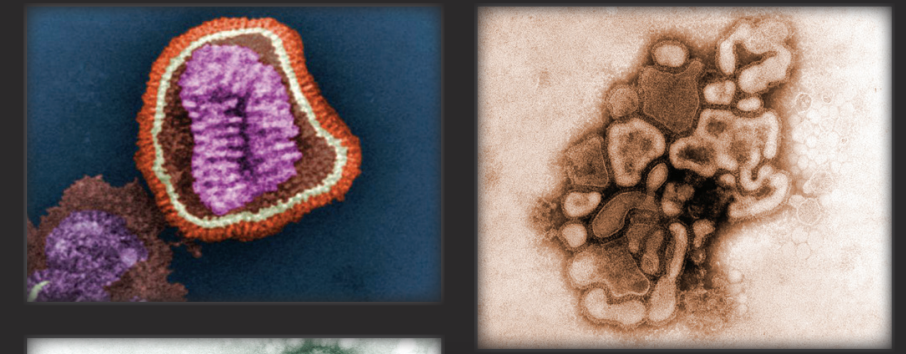 \\ The Public Health Image Library (PHIL), Centers for Disease Control and Prevention, contains thousands of public
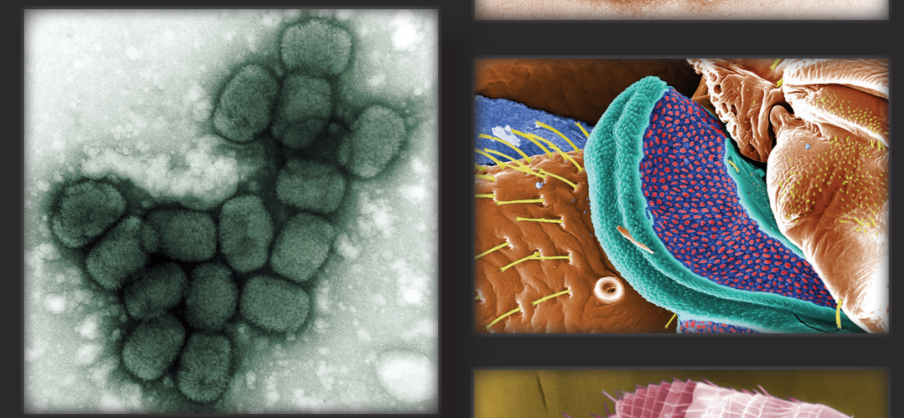 health-related images, including high- resolution (print quality) photographs, illustrations, and videos. \\ PHIL collections illustrate
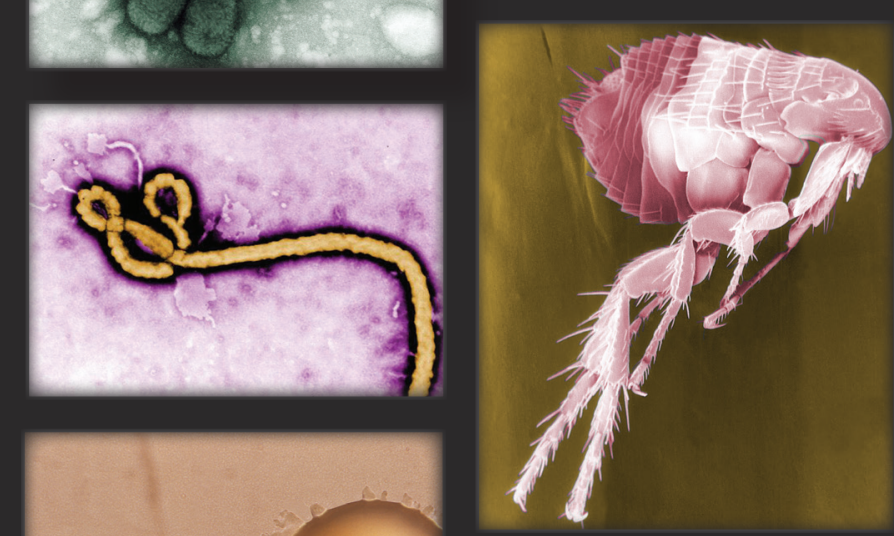 current events and articles, supply visual content for health promotion brochures, document the effects of disease, and enhance instructional media.

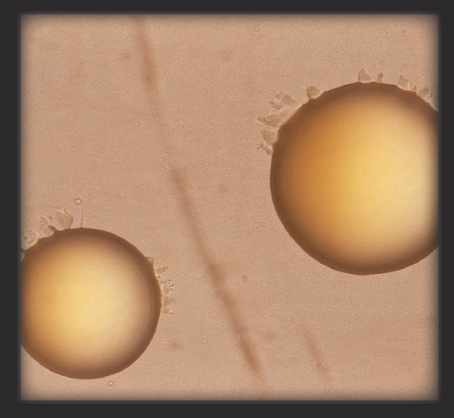 \\ PHIL images, accessible to $\mathrm{PC}$ and Macintosh users, are in the public domain and

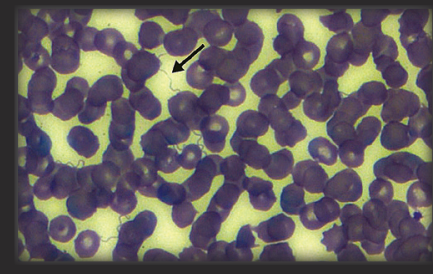 available without charge. \\ Visit PHIL at: http: / /phil.cdc.gov/phil}

